\title{
Distillation of Posterior Fossa Demyelination in Acute Vestibular Syndrome: the Eyes Have It
}

\author{
Aasef G. Shaikh ${ }^{1}$ Mario Manto ${ }^{2}$ \\ Published online: 27 April 2019 \\ (C) Springer Science+Business Media, LLC, part of Springer Nature 2019
}

\begin{abstract}
Separating the etiologies of an acute vestibular syndrome (AVS) of central origin is a clinical challenge; the common causes include (1) stroke of the brainstem/cerebellum and (2) demyelinating disorders such as multiple sclerosis (MS) and neuromyelitis optica spectrum disorder (NMOSD). Overshadowed by the vascular etiologies, the literature describing AVS due to demyelinating disorders has been growing through the last decade. The discovery of IgG-NMO, a specific pathogenic antibody directed against the astrocytic water channel aquaporin-4 (AQP4), has improved the differential diagnoses between MS and NMOSD. AQP4 is particularly expressed in ependymal/subependymal astrocytes and glia limitans astrocyte processes, including around the fourth ventricle. Adding a clinical biomarker to distinguish MS and NMOSD in AVS patients, as reported in this issue, will be of great clinical value.
\end{abstract}

Sudden damage to the peripheral balancing apparatus or the vestibular nerve conducting the sensory information from the vestibular labyrinth to the brainstem results in a sudden onset of continuous vertigo, intolerance to head movements, along with intractable nausea and vomiting. About two decades ago, Hotson and Baloh coined the term acute vestibular syndrome (AVS) describing this well-known phenomenon [1]. Commonly caused by the damage to the vestibular nerve due to the unknown source of inflammation, called vestibular neuritis, the estimated incidence of the AVS is 3.5 per 100,000 people. One out of five of dizzy patients visiting the emergency department has an AVS [2]. The cerebellar or brainstem ischemia causes this phenomenon in about $5-10 \%$ of patients [3]. Frequently overshadowed by neuritis or stroke, the AVS can be caused by multiple sclerosis (MS), a common immune condition. Approximately 4\% of the AVS patients were thought to have a demyelinating disease affecting the cerebellum or the brainstem; the incidence is not much lower compared to that presented with stroke [4].

Aasef G. Shaikh

aasefshaikh@gmail.com

1 University Hospitals and Cleveland VA Medical Center, Case Western Reserve University, 11100 Euclid Avenue, Cleveland, OH 44110, USA

2 Chef du Service de Neurologie, CHU-Charleroi, Charleroi, Belgium
About 45 years ago, the first ever published description of the AVS in demyelinating disorder appeared in the German literature [5]. Subsequently, a handful of case reports supported the idea that the new lesions in MS or exacerbation of previously existing plaques in areas of brainstem or cerebellum can present with intractable vertigo, nausea, and vomiting [6-8]. Within the last decade, there was a rapid growth in the literature reporting demyelinating disorders as a cause of AVS. In one study, out of 1153 patients who had MS and vertigo, 25 were evaluated during the acute spell. One-third of these patients were identified to have the demyelinating disorder as a cause of acute vestibular presentation [9]. A subsequent study examining the epidemiology in 187 patients who presented with acute onset of vertigo [10] had a rather low incidence of MS $(\sim 0.5 \%)$. Such disparity in the frequency could be because the latter study was performed in Shanghai (China) where there is up to $30 \times$ lower prevalence of MS (1-2 per 100,000) compared to its global prevalence $(33$ per 100,000) $[11,12]$. The subsequent literature from the Americas presented a prospective observational study over 12 years; studying 170 patients with AVS. About $4 \%$ of these patients had demyelinating disease; all had central ocular motor signs with two had normal head impulse test (HIT, a widely used clinical assessment technique involving the assessment of horizontal semicircular canal and superior vestibular nerve function in response to small amplitude $\sim 10^{\circ}$ and high acceleration $3000-4000^{\circ} / \mathrm{s}^{2}$ rotational head impulses measuring the angular vestibulo-ocular reflex). All had intractable vertigo with nystagmus, nausea, vomiting, intolerance to head movement, and unsteadiness [4]. 
The subsequent study reported that approximately $16 \%$ of patients who visited the emergency room for "imbalance" and $10 \%$ of patients who came to the emergency room for "double vision and vertigo" were diagnosed with MS. The reported literature emphasized neuroimaging with some focus on clinical signs such as the presence of nystagmus, abnormal HIT, skew deviation, and cerebellar motor deficits, i.e., gait dysfunction and dysarthria. It is well-known that demyelinating disorders present in various flavors. Optimal therapeutic response is often dictated by the underlying subtype of the demyelinating disease. Finally, demyelinating disorders are also segregated by the underlying pathophysiology. It is, however, unclear whether the specific subtype of the posterior fossa demyelinating disorders, such as neuromyelitis optica spectrum disorder (NMOSD) versus MS causing an AVS, favors peculiar ocular motor phenomenology. Such phenotypic classification improving the clinical diagnostic accuracy is challenging as it requires objective assessments in a large number of patients; heavily powered investigations are uncommon in ocular motor field due to relatively rare incidence of eye movement disorders.

In an elegant, extensive, and rigorously performed study, Lee and colleagues [13] dissected the vestibular and ocular motor presentation of the demyelinating disorder asking a question whether this constellation of findings allow an opportunity to classify them among different subtypes. The authors studied 68 patients with demyelinating disorders presenting with vertigo, 42 had the diagnosis of MS involving the cerebellum, and 26 patients had NMOSD, of all 41 patients presented with the AVS. There was a unique pattern of ocular motor deficits - those with NMOSD invariably had horizontal gaze-evoked nystagmus; not consistently seen in those with cerebellar MS. Abnormal HIT was also common in those with NMOSD; the key distinguishing feature was that abnormal horizontal HIT was either bilateral or if unilateral it was not specific to the ipsilateral or contralateral lesion. This contrasted with abnormal HIT in cerebellar MS - inconsistent and when present was always abnormal ipsilateral to the side of the lesion. Nausea and vomiting were frequent in NMOSD. Translating these findings to clinical diagnostic specificity - a combination of horizontal gaze-evoked nystagmus and bilaterally abnormal HIT, when presents with AVS, likely suggests a possibility of NMOSD. The knowledge of such ocular motor presentation profile may not necessarily offer a stand-alone diagnostic marker all the times. Nevertheless, peculiar eye movement deficits present in combination with other neurological deficits can improve the diagnostic accuracy. Laboratory tests measuring the levels of aquaporin antibodies (anti-AQP4) takes several days to weeks; MRI can be effective, but small lesions in posterior fossa are often not visible in MRI. Most importantly, conjunction of MRI with clinical discrimination ability tremendously improves our diagnostic accuracy.
Distinguished ocular motor features that specifically characterize NMOSD also explain their mechanistic underpinnings. The anti-AQP4 antibodies are frequently present in the region of the third and fourth ventricles - the area where there are abundant vestibular and eye movement sensitive neurons clustered in the vestibular nucleus and prepositus hypoglossi. Autoimmune attack on these neurons physiologically explains the consistent occurrence of gaze-evoked nystagmus, a characteristic feature of horizontal ocular motor neural integrator dysfunction. Area postrema, an emesis center, is also in the vicinity of the third and fourth ventricle explaining frequent nausea. When an immune attack involves the vestibular nerve fibers or the direct pathway for the vestibular ocular reflex - the three neuron arc comprised of vestibular afferents, connection of vestibular nuclei to the ocular motor nuclei, and ocular motoneuron, again in the area of the third and fourth ventricles; the abnormal HIT is seen. Damage to the midline internuclear fibers would lead to bilateral dysfunction, damage to vestibular nerve fascicle (first-order neuron of the three neuron arc) leads to unilateral dysfunction, while impairment in the ocular motor fascicle (third-order neuron of the three neuron arc) leads to contralateral vestibular ocular reflex dysfunction.

In summary, we have now strong evidence that eye movements often succeed as the reliable markers distinguishing acute neurological disorders, frequently needing prompt therapeutic interventions. The examination of eye movements is straightforward, not requiring any technical infrastructure, and their constellations frequently link with specific disease localization. In conjunction with other modalities, such as imaging or laboratory investigations, the eye movement examination makes the diagnostic accuracy only better.

\section{Compliance with Ethical Standards}

Conflict of Interest The authors declare that they have no conflict of interest.

\section{References}

1. Hotson JR, Baloh RW. Acute vestibular syndrome. N Engl J Med. 1998;339(10):680-5.

2. Tarnutzer AA, Berkowitz AL, Robinson KA, Hsieh YH, NewmanToker DE. Does my dizzy patient have a stroke? A systematic review of bedside diagnosis in acute vestibular syndrome. CMAJ. 2011;183(9):E571-92.

3. Newman-Toker DE. Missed stroke in acute vertigo and dizziness: it is time for action, not debate. Ann Neurol. 2016;79(1):27-31.

4. Pula JH, Newman-Toker DE, Kattah JC. Multiple sclerosis as a cause of the acute vestibular syndrome. J Neurol. 2013;260(6): 1649-54.

5. Gottwald W. Paroxysmal syndromes in multiple sclerosis (author's transl). MMW Munch Med Wochenschr. 1976;118(31):983-6. 
6. Schroth WS, Tenner SM, Rappaport BA, Mani R. Multiple sclerosis as a cause of atrial fibrillation and electrocardiographic changes. Arch Neurol. 1992;49(4):422-4.

7. Hasegawa $\mathrm{H}$, et al. Acute relapsing disseminated encephalomyelitis (ARDEM) mimicking a temporal lobe tumor. No Shinkei Geka. 1994;22(2):185-8.

8. Sasaki O, Ootsuka K, Taguchi K, Kikukawa M. Multiple sclerosis presented acute hearing loss and vertigo. ORL J Otorhinolaryngol Relat Spec. 1994;56(1):55-9.

9. Frohman EM, Zhang H, Dewey RB, Hawker KS, Racke MK, Frohman TC. Vertigo in MS: utility of positional and particle repositioning maneuvers. Neurology. 2000;55(10):1566-9.

10. Zhang Y, Chen X, Wang X, Cao L, Dong Z, Zhen J, et al. A clinical epidemiological study in 187 patients with vertigo. Cell Biochem Biophys. 2011;59(2):109-12.
11. Cheong WL, Mohan D, Warren N, Reidpath DD. Multiple sclerosis in the Asia Pacific region: a systematic review of a neglected neurological disease. Front Neurol. 2018;9:432.

12. Cheng Q, Cheng XJ, Jiang GX. Multiple sclerosis in China-history and future. Mult Scler. 2009;15(6):655-60.

13. Lee SU, Kim HJ, Choi JH, Choi JY, Kim JS. Comparison of ocular motor findings between neuromyelitis optica spectrum disorder and multiple sclerosis involving the brainstem and cerebellum. The Cerebellum. 2019;18(3):511-18.

Publisher's Note Springer Nature remains neutral with regard to jurisdictional claims in published maps and institutional affiliations. 